\title{
Automated analysis of contractility in the embryonic stem cell test, a novel approach to assess embryotoxicity
}

\author{
Annelieke K. Peters ${ }^{\mathrm{a}, *}$, Gert Van de Wouwer $^{\mathrm{b}}$, Barbara Weyn ${ }^{\mathrm{b}}$, Geert R. Verheyen ${ }^{\mathrm{b}}$, \\ Philippe Vanparys ${ }^{\mathrm{a}, 1}$, Jacques Van Gompel ${ }^{\mathrm{a}}$ \\ a JohnsonEJohnson Pharmaceutical RED (JEJ-PRD), Department of Genetic and Exploratory Toxicology, Turnhoutseweg 30, B-2340 Beerse, Belgium
}

${ }^{\mathrm{b}}$ Digital Cell Imaging Labs (DCI), Sorbrechtshofstraat 5, B-2650 Edegem, Belgium

\section{A R T I C L E I N F O}

\section{Article history:}

Received 4 July 2008

Accepted 12 September 2008

Available online 19 September 2008

\section{Keywords}

Embryonic stem cells

D3

EST

High throughput

Automated

Image analysis

\begin{abstract}
A B S T R A C T
The embryonic stem cell test (EST) is an ECVAM-validated assay to detect embryotoxicity. The output of the assay is the effect of test compounds on the differentiation of murine-derived embryonic stem cells (D3 cells), recorded by visual analysis of contracting cardiomyocyte-like cells. Incorporation of a system to assess the contractility in an automated manner is proposed, to increase the throughput in the EST independent of observer bias. The automated system is based on image recording of each well, resulting in the area (pixels) and frequency of contractility $(\mathrm{Hz})$. Four test compounds were assessed for their embryotoxic potency in the 96-well version of the EST, with both manual and automated analysis: 6Aminonicotinamide, Valproic Acid, Boric Acid, and Penicillin G. There was no statistically significant difference in the outcome of both methods in the fraction of contractility $(p<0.05)$, resulting in the same rank-order of Relative Embryotoxic Potency (REP) values: 6-aminonicotinamide (1) > valproic acid (0.007-0.013) > Boric Acid (0.002-0.005) > Penicillin G (0.00001). The automated image recording of contractile cardiomyocyte-like cells in the EST allows for an unbiased high throughput method to assess the embryotoxic potency of test compounds, resulting in an outcome comparable to manual analysis.
\end{abstract}

(c) 2008 Published by Elsevier Ltd.

\section{Introduction}

Throughout the gestational period, embryos are susceptible to xenobiotics. Adverse reactions to chemicals or pharmaceutical compounds could result in developmental toxicity. An example is Thalidomide, a compound that was used extensively among pregnant women in the 1950s, though withdrawn from the market after observations of its teratogenic potency. Therefore, testing chemical compounds and pharmaceuticals for their embryotoxic potential is of importance.

Existing in vivo tests, based on maternal or embryonic exposure of laboratory animals, are time-consuming and expensive. An alternative to the in vivo assessment is offered by in vitro assays like the whole-embryo culture assay (WEC test) and the micromass assay (MM), although both still rely on embryos (Piersma et al., 1995; Spielmann et al., 1997; Genschow et al., 2002; Piersma, 2004). The embryonic stem cell test (EST) however, is an in vitro tool to assess the developmental toxic potency of test compounds in early development (Spielmann et al., 1997). This method has been validated by the European Centre for the Validation of Alternative

\footnotetext{
* Corresponding author. Tel.: +32 14608329; fax: +32 14605150 .

E-mail address: APeters5@prdbe.jnj.com (A.K. Peters).

1 Current address: Altoxicon BVBA, Boskant 101, B-2350 Vosselaar, Belgium.
}

Methods (ECVAM) (Genschow et al., 2004). Embryonic stem cells can differentiate into all cell types of the three germ layers: ectodermal, mesodermal, and endodermal. As a result, a culture of differentiated stem cells will consist of a heterogeneous population with cells from various lineages (reviewed by (Rohwedel et al., 2001). A controlled differentiation into one of these lineages can be used to assess interference of test compounds with these processes.

The EST is based on the formation of embryonic bodies (EBs) when murine-derived embryonic stem cells (D3 cells) are placed in a 'hanging drop' culture. When subsequently seeded in tissue culture plates, the cardiomyocyte lineage is the predominant differentiation route of the cells, resulting in contractile areas in the EBs. In the EST, inhibition of differentiation of the cells into contractile cardiomyocytes is manually recorded by microscopic analysis, as an in vitro measurement of developmental toxicity.

Previously, we have made adaptations to the experimental procedure of the EST to optimise the differentiation potential and ensure a higher throughput of the assay (Peters et al., 2007, 2008; De Smedt et al., 2008). Implementation of a low attachment 96-well plate with subsequent seeding of the EBs into a tissue culture coated 96-well plate increased the maximum number of test compounds. Furthermore, the 96 -well format greatly reduced the costs involved since it requires only about $15 \%$ of the cell culture medium and test compounds compared to the ECVAM-validated 24-well EST (range-finder and two repeat studies). 
Table 1

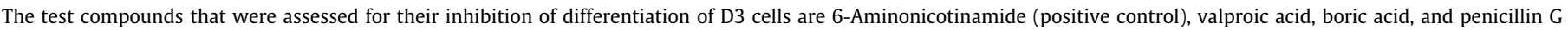<smiles>NC(=O)c1ccc(N)nc1</smiles>

6-Aminonicotinamide

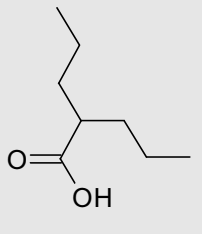<smiles>OB(O)O</smiles>

Boric acid

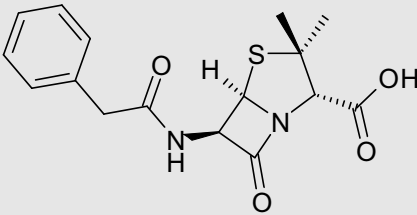

Penicillin G
However, the manual assessment of contractility remained the quantity-limiting step when multiple compounds were tested for their embryotoxic potential. Visual examination of contractility by microscopic analysis is labour-intensive, and could result in a technician-dependent outcome. Furthermore, the observed contractility is noted in a binominal 'yes/no contractility observed', without taking the variability in the contractile frequency or size of the contractile area of the EB into account.

Recent modifications to the EST include addition of various endpoints, such as molecular markers or FACS analysis (Bremer et al., 2001; Seiler et al., 2004; Zur Nieden et al., 2004; Adler et al., 2005, 2008; Honda et al., 2006). These modifications result in a more quantitative output than visual examination, but are not suitable for the desired high-throughput assessment. Accordingly, there is a desire to develop a method to automate the assessment of contractility, facilitating the evaluation without possible observer bias.

Walmod and colleagues have reported an automated in vitro screening of teratogens (Walmod et al., 2002). Based on staining of murine-derived fibroblasts (L929 cells), automated computerized video recording and detection was used to identify possible teratogens. With their system, 20 compounds were assessed for the teratogenic potency based on computerized recognition of changes in cell morphology and cell proliferation (Walmod et al., 2004). A different approach of automated screening for developmental toxicity is the quantification of the contractility of the embryonic stem cells. Contractility of cardiomyocytes or calcium fluctuations have been assessed with confocal laser-scanning microscopy or detection of fluorescence dyes (Sauer et al., 1998; Satoh et al., 2000; Dolnikov et al., 2006). However, the manner in which the EBs contract is not taken into account with these methods, while there can be large differences in both their size and frequency of contraction.

The objective of the present study is to ensure technician-independent and unbiased outcome of the EST by automated assessment of the contractility of embryonic stem cells in a 96-well format. The following test compounds were chosen to be assessed both manually and automated: 6-Aminonicotinamide (positive control), Boric acid, Valproic acid, and Penicillin G (Table 1). The selection of test compounds was based upon available literature (Smith et al., 1983; Schwetz and Harris, 1993; Spielmann et al., 2001; Brown, 2002; Genschow et al., 2004) and previously reported outcomes (Peters et al., 2008).

\section{Material and methods}

\subsection{Chemicals}

The chemicals used in the studies were obtained from the following companies: DMSO was purchased from Merck (VWR; Leuven, Belgium). Dulbecco's Modified Eagle's Medium (DMEM supplemented with L-glutamine, glucose and $\mathrm{NaHCO}_{3}$ ), L-glutamine, non-essential amino acids (NEAA), and phosphate buffered saline (PBS) without $\mathrm{Ca}^{2+}$ and $\mathrm{Mg}^{2+}$ were ordered from Gibco (Carlsbad, CA, USA). Foetal Calf Serum (FCS; stem cell tested) was ordered from Hyclone (Perbio Science; Erembodegem-Aalst Belgium), mLIF (ESGRO ${ }^{\circledR} 10^{6} \mathrm{U}$ ) from Chemicon (Millipore; Brussels, Belgium), and Penicillin/Streptomycin solution (5000 U/ $5000 \mu \mathrm{g}$ ) from Cambrex (VWR; Leuven, Belgium). All other chemicals were ordered at Sigma Aldrich (St. Louis, MO, USA) and dissolved in DMSO (final solvent concentration $0.25 \%$ ).

\subsection{Cell line and cell culture}

Murine-derived embryonic stem cells (D3; Mus Musculus) were kindly provided by ECVAM (Ispra, Italy), by courtesy of S. Bremer. The D3 cells were cultured in DMEM supplemented with $20 \%$ heat inactivated FCS, $2 \mathrm{mM}$ L-glutamine, $50 \mathrm{U} / \mathrm{ml}$ penicillin, $50 \mu \mathrm{g} / \mathrm{ml}$ streptomycin, $1 \% \mathrm{NEAA}$, and $0.1 \mathrm{mM} \beta$-mercaptoethanol. For routine culture, $1000 \mathrm{U} / \mathrm{ml} \mathrm{mLIF}$ was added directly to the medium after seeding the cells. The cells were cultured in a humidified atmosphere $\left(5 \% \mathrm{CO}_{2}, 37^{\circ} \mathrm{C}\right)$, and routinely subcultured.

\subsection{Embryonic stem cell test}

The EST was performed as described previously (Peters et al., 2008). In short, D3 cells were seeded in culture medium in round bottom low attachment 96-well plates (300 cells/well in $50 \mu \mathrm{l}$; NUNC round bottom low attachment well plates ref. 145399). Three days later, $50 \mu \mathrm{l}$ fresh medium with test compounds was added.

The embryonic bodies (EBs) formed during the first five days of the experiment, were transferred into flat bottom tissue culture 96-well plates, with addition of fresh medium with test compounds. After all the EBs were transferred, the tissue culture treated well plate was placed on a digital orbital mini-shaker with microtitre plate insert to ensure a central position of the EB in the well (IKA MS3, Staufen, Germany; $\pm 250 \mathrm{rpm}$ during approximately $40 \mathrm{sec})$, and subsequently placed in the incubator $(5 \%$ $\left.\mathrm{CO}_{2}, 37^{\circ} \mathrm{C}\right)$. At the tenth day of the experiment, the contractility of the cells was assessed visually (Zeiss Axiovert 200,100X), and recorded as '+' (contractile areas observed) or '-' (no contractile areas observed).

\subsection{Statistical Analysis of the EST}

Statistical analysis of the dose-response curves from the EST was performed using GraphPad Prism version 4.00. After an initial range-finding experiment, the EST was carried out in two independent experiments (10 EBs/concentration) and expressed as 'fraction contractile EBs', with $100 \%$ implying all EBs exposed to the same concentration of test compound resulted in contractile areas 
in the EBs. The in vitro relative embryotoxic potency (REP) was calculated relative to the $\mathrm{EC}_{50}$ value obtained for the positive control (6-Aminonicotinamide) of each independent experiment in 'fraction contractility' (Eq. (1) ). The $\mathrm{EC}_{50}$ is the concentration where $50 \%$ of the maximally observed effect was reached. When no $\mathrm{EC}_{50}$ value could be determined (no inhibition of differentiation), or when there was no statistically significant difference to the observed decrease in cell viability, the REP was given an arbitrary value of 0.00001 .

$\frac{\mathrm{EC}_{50} \text { value (positive control } 6 \mathrm{AN} \text { ) }}{\mathrm{EC}_{50} \text { value (test compound) }}=\mathrm{REP}$ value (test compound)

\subsection{Automated assessment of contractility}

The image analysis system consists of an Axiovert S100 inverted microscope (Zeiss, Oberkochen, Germany) with Kohler illumination (condenser $N A=0.55$ ) equipped with a motorized $(x, y)$-stage (Märzhauser, Wetzlar, Germany), focus-drive and a Firewire monochrome CCD-camera (Dolphin F-145B, Allied Vision Technologies, Stadtroda, Germany). The UGR software (Digital Cell Imaging Labs, Edegem, Belgium) running on a standard PC (Dell Precision Workstation with Microsoft Windows XP) was used to control both stage and camera.

The detection of the embryonic body occurred manually by moving the stage to the centre of the embryonic body, followed by an auto-focus step, and subsequent time-lapse image recording. The recordings were taken with brightfield imaging settings, illuminated with a halogen lamp over a time-span of $9 \mathrm{~s}$, using a frame rate of 3.33 frames/second in greyscale (see Fig. 1). The resolution of the recorded image was $1.05 \mu \mathrm{m} /$ pixel, i.e. 1 pixel represents approx. $1.05 \mu \mathrm{m}^{2}$. Image analysis was performed using the Beat Assay Analysis (BEA) software (Digital Cell Imaging Labs, Edegem, Belgium). The software detects movement in cells by comparing each time frame to the previous and next frames of the video sequence. The area of contractile cells is computed at each time point. In some cases, false positive contractile cells were detected at the end or beginning of the movie (Fig. 1), as confirmed by visual examination. This was due to border effects, i.e. not having frames prior or after the particular frame that was being analysed. The border effects were avoided by discarding the first and last frames of the compiled movie from the analysis.

The following features were computed with the BEA software for each time point: the contractile area of the EB for each individual well (in pixels, Fig. 1), and the frequency of the contractions of the EB (in Hz, Fig. 2).

\subsection{Statistical Analysis of the automated assessment of contractility}

The image analysis data was presented as contractile area (pixels) and frequency of contractility ( $\mathrm{Hz})$. Based on the obtained data for the area of contractility, the automated image analysis was also presented in 'fraction contractility'. To convert the data into 'fraction contractility', thresholds were set for empty wells and for wells containing non-contractile EBs. The outcome of a total of 50 empty wells (data computed for the contractile area) was compiled, and based upon the computed data, trial and error resulted in a threshold of 1500 pixels for empty wells. In a similar way, the threshold for wells containing non-contractile EBs was set at 5,5000 pixels.

After these thresholds were set, the outcome of the automated image analysis was converted into 'fraction contractility'. Comparison between the manual and automated data sets (both in 'fraction contractility') was performed with an extra sum-of-squares F-test ( $p<0.05$; GraphPad Software, San Diego, CA, USA).

\subsection{Cell viability}

For the cell viability determination, cells were seeded at a density of 1000 cells/well in a 96-well plate (tissue culture coated) and exposed to test compounds two hours later. Medium with test compounds was refreshed three days later, and after a six day incubation period, replaced with a $0.5 \mathrm{mg} / \mathrm{ml}$ MTT (3-(4,5-dimethylthiazol-2-yl)-2, 5-diphenyltetrazolium bromide) solution (60 min) as described previously (Peters et al., 2008). Subsequently, the plates were spun ( $1500 \mathrm{~g}, 5 \mathrm{~min}$ ) and formazan was extracted from the

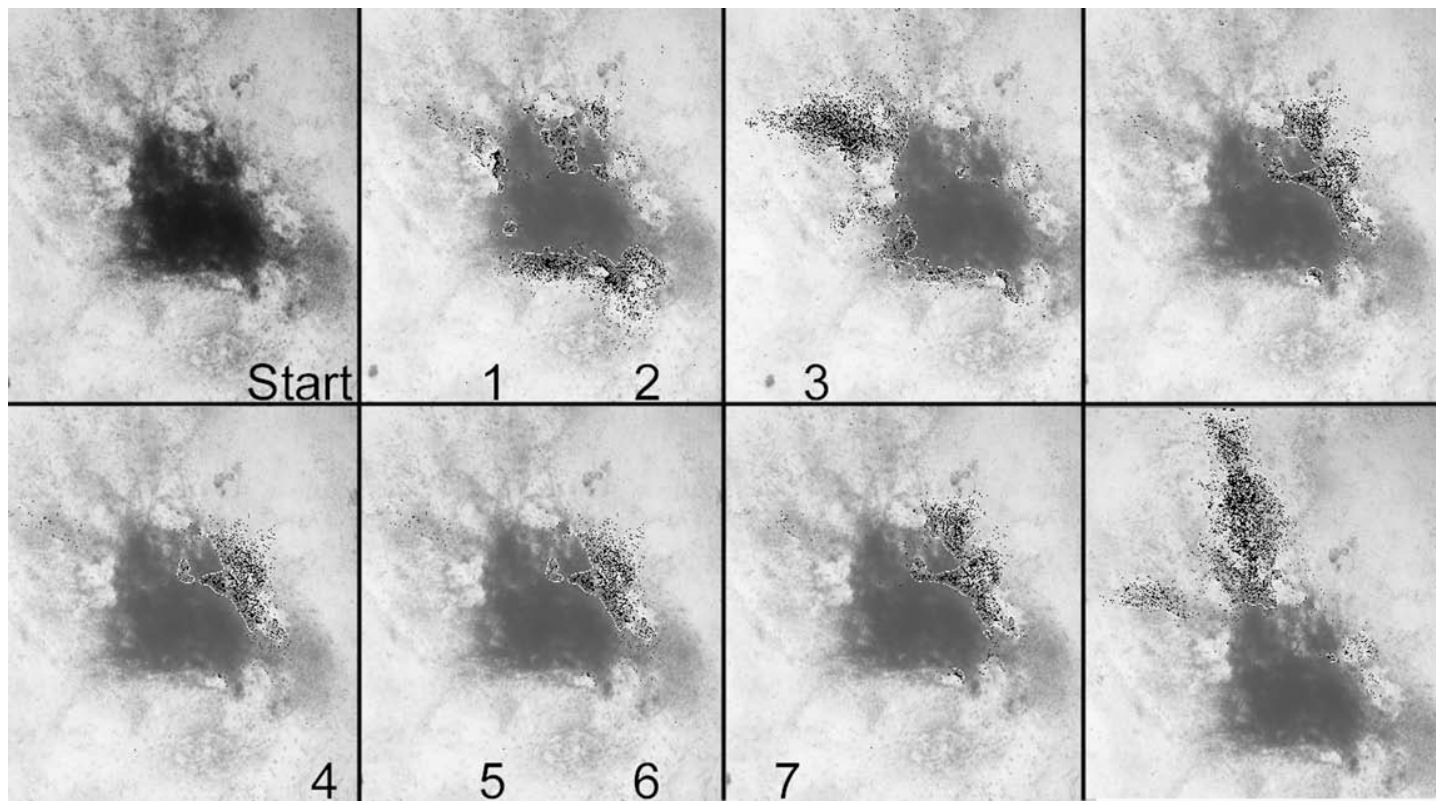

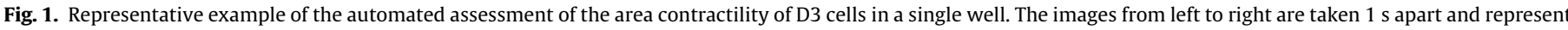

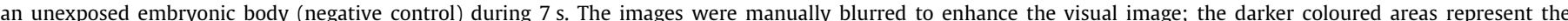

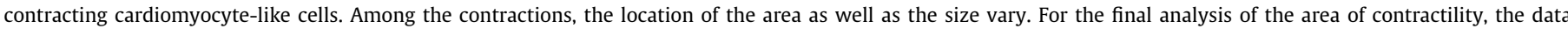
represented in the Figure was used from second 3 until second 6 (with 3.33 frames/second), to compensate for boundary effects at the beginning and end of the movie. 

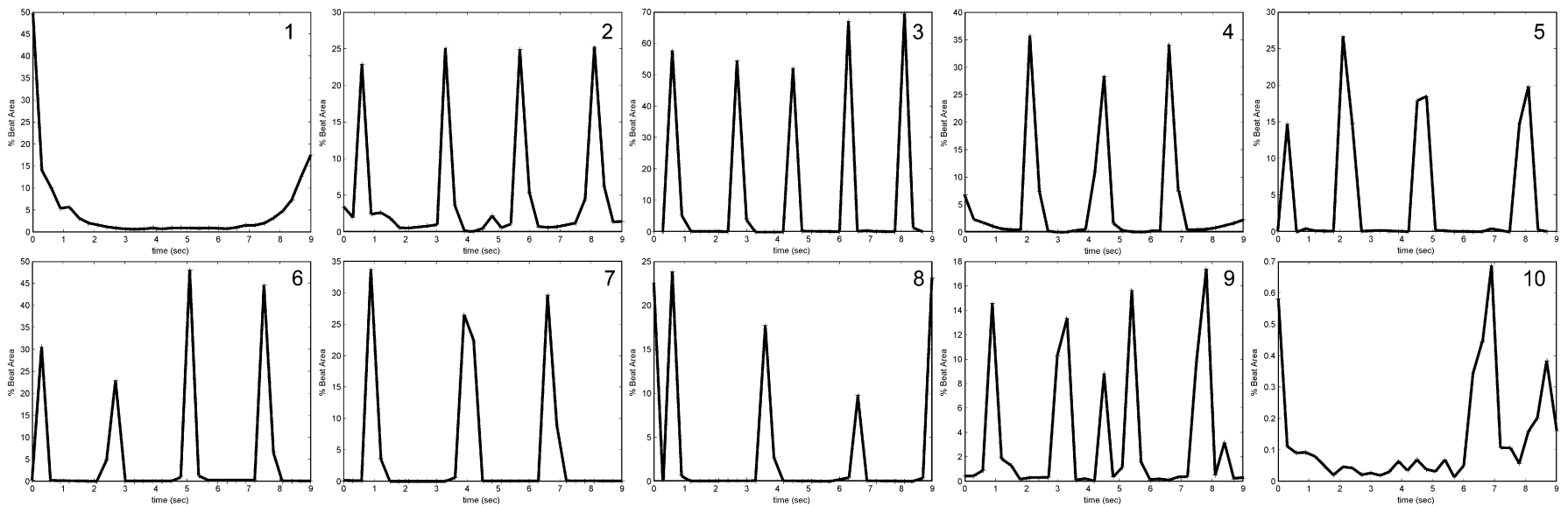

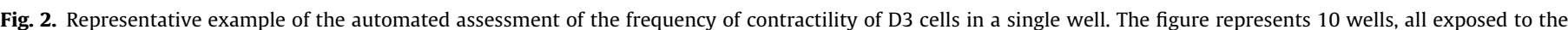

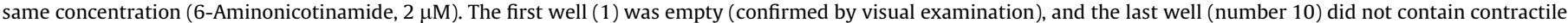

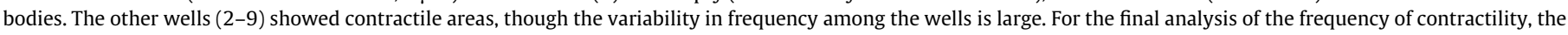

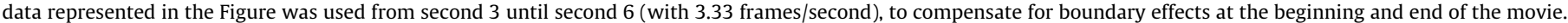

cells with $100 \mu \mathrm{l}$ DMSO/well (15 min shaking at $200 \mathrm{rpm}$ ). The concentration of formazan was determined spectrophotometrically at $570 \mathrm{~nm}$. The assay was performed in three independent experiments with exposure to the test compounds in 6-fold.

\subsection{Statistical Analysis of the cell viability assay}

Statistical analysis of the cell viability assay was performed using GraphPad Prism version 4.00. In order to determine whether the observed effects on the differentiation of the stem cells into the cardiomyocyte-like cells were due to embryotoxicity or cytotoxicity of the test compounds, the dose-response curves of both assays were compared to one-another with an extra sum-of-squares Ftest ( $p<0.05$; GraphPad Software, San Diego, CA, USA). When no difference between the outcome of the cell viability assay and the differentiation assay could be observed, the REP was given an arbitrary value of 0.00001 (Peters et al., 2007).

\section{Results}

Previously, the developmental toxic potency of twelve test compounds was assessed in the 96-well EST format. The outcome of the EST was compared to the outcome of the cell viability assay in order to differentiate embryotoxicity from cytotoxicity (extra sum-of-squares F-test, $p<0.05$ ). The concentrations of the compounds used in the current study were based on these previous findings, avoiding a cytotoxic range. In Table 2 , the $\mathrm{EC}_{50}$ values from the cell viability assay (when detectable) are presented.

\subsection{Embryonic stem cell test - manual assessment of contractility}

Exposure of the cells to the test compounds 6-Aminonicotinamide (maximum concentration $1.5 \mu \mathrm{M}$ ), Boric acid (maximum concentration $4.8 \mathrm{mM}$ ), and Valproic Acid (maximum concentration $1.7 \mathrm{mM}$ ) resulted in a dose-response relationship when the outcome of the EST was assessed manually (Figs. 3-5A). Exposure of the cells to the negative control Penicillin $G$ (maximum concentration $3 \mathrm{mM}$ ) did not result in a dose-response relationship after manual assessment of contractility (Fig. 6A), the EBs retained their contractile capacity. In Table 2 , the $\mathrm{EC}_{50}$ values $(\mu \mathrm{M})$ and corresponding Relative Embryotoxic Potency ( $\mathrm{REP}_{\text {manual }}$ ) values are presented (left panel). When the compounds are ranked according to their REP manual value, the following order was obtained: 6-Aminonicotinamide (1) > Valproic Acid (0.007-0.007) > Boric acid (0.005$0.002)>$ Penicillin $G(0.00001)$.

\subsection{Embryonic stem cell test - automated assessment of contractility}

Automated image analysis of the contractile cells is presented as 'contractile area' (Figs. 3-6B) and 'frequency of contractility (Figs. 3-6C). The automated computed data of the area and frequency of the contractions resulted in a large variation. Embryonic bodies that were close to the side of the well did not pose a problem for the image analysis of the contractile frequency. However, the contractile area could not be correctly computed, since the EBs tended to grow upward and sideward, following the well lining. This effect was taken into account when thresholds were set

Table 2

The inhibition of differentiation of D3 cells into cardiomyocytes after exposure to test compounds was assessed manually (on the left) and automated (on the right)

\begin{tabular}{|c|c|c|c|c|c|}
\hline \multirow[t]{2}{*}{ Compound } & \multicolumn{2}{|c|}{ Manual assessment of contractility } & \multicolumn{2}{|c|}{ Automated assessment of contractility } & \multirow{2}{*}{$\frac{\text { Cell viability }}{\mathrm{EC}_{50}(\mu \mathrm{M})}$} \\
\hline & $\mathrm{EC}_{50}(\mu \mathrm{M})$ & REP value ${ }^{\S}$ & $\mathrm{EC}_{50}(\mu \mathrm{M})$ & REP value ${ }^{\S}$ & \\
\hline & run 1 -run 2 & run 1 -run 2 & run 1 -run 2 & run 1 -run 2 & \\
\hline 6-Aminonicotinamide (6AN) & $5.92-3.55$ & $1-1^{\mathrm{a}}$ & $5.352-3.5$ & $1-1$ & $19.7 \pm 6.0$ \\
\hline Valproic acid & $867.3-523.2$ & $0.007-0.007^{\mathrm{a}}$ & $709.2-268.9$ & $0.008-0.013$ & $1756.3 \pm 392$ \\
\hline Boric acid & $1291-1587$ & $0.005-0.002^{\mathrm{a}}$ & 1196-1672 & $0.0045-0.0021$ & $2226.0 \pm 545$ \\
\hline Penicillin G & nd-nd & $0.00001-0.00001^{*}$ & nd-nd & $0.00001-0.00001^{*}$ & nd \\
\hline
\end{tabular}

The data are presented as $\mathrm{EC}_{50}$ of the fraction contractility (\%), and relative embryotoxic potency (REP) value from two individual experiments.

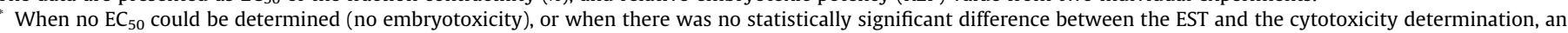
arbitrary REP value of 0.00001 was given.

a There is no statistically significant difference between the manual and automatic method ( $p<0.05$, extra-sum-of-squares F-test). Ranking the compounds according to

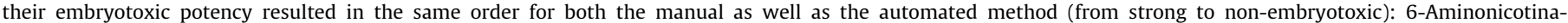
mide $>$ Valproic acid > Boric acid > Penicillin G (nd: not detectable). 


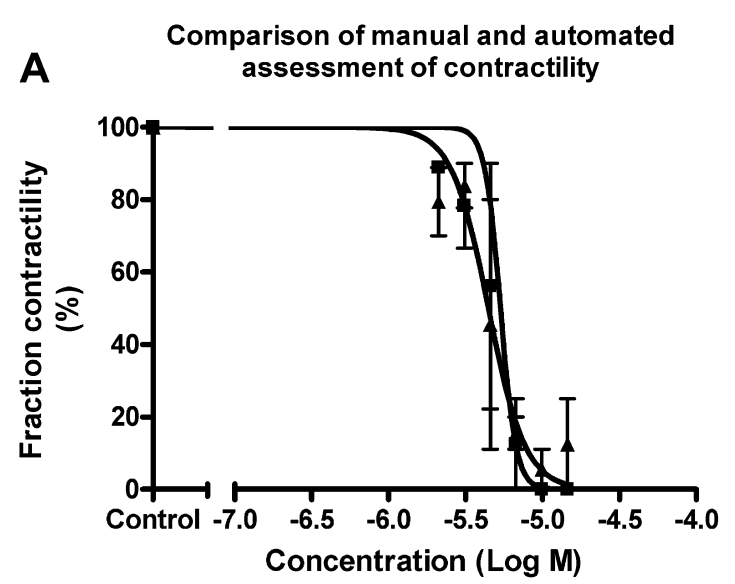

- 6-Aminonicotinamide manual assessment

\ 6-Aminonicotinamide automated assessment

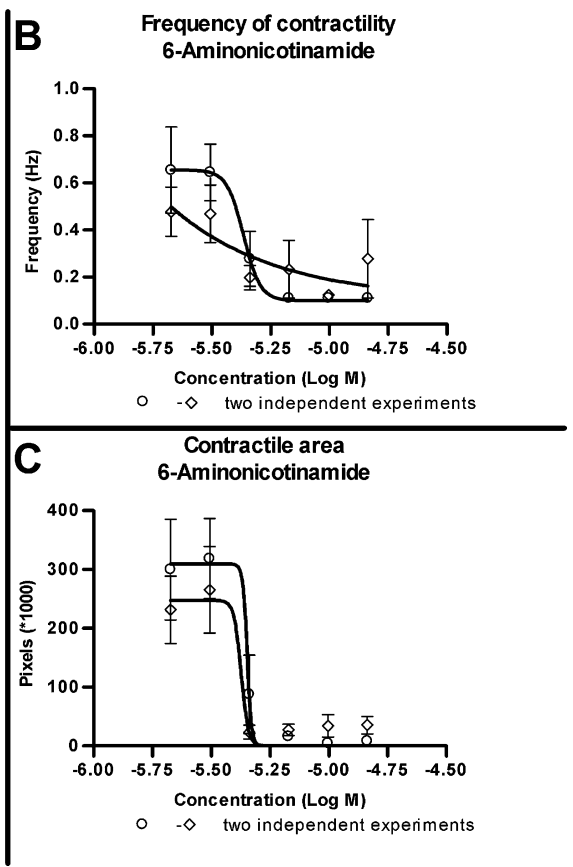

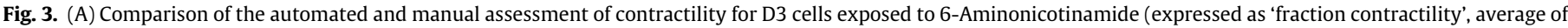

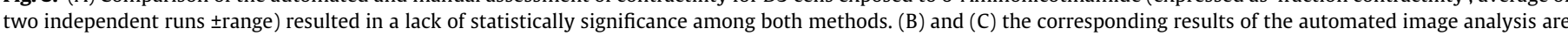

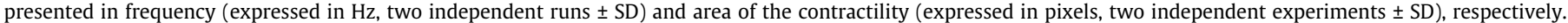

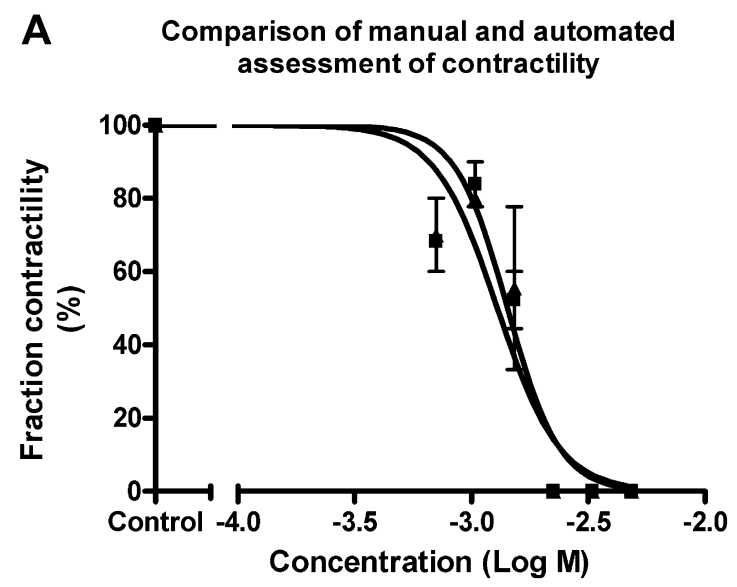

- Boric Acid manual assessment

- Boric Acid automated assessment

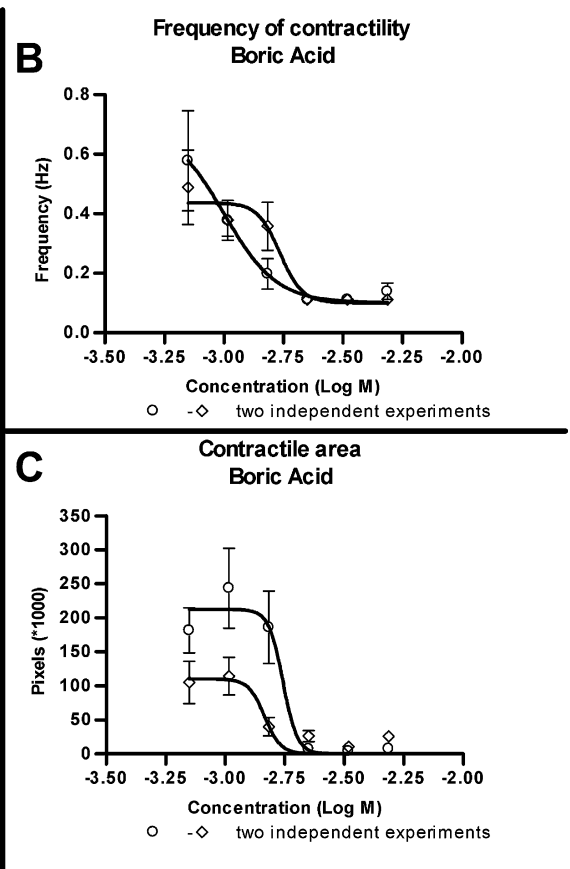

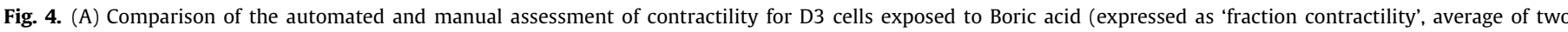

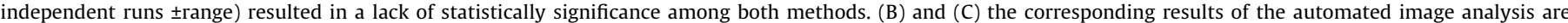

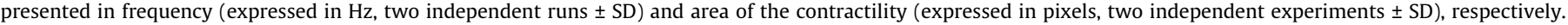

to convert the data into 'fraction contractility': $<1500$ pixels represents an empty well, $1500>$ pixels $<55,000$ represents a non-contractile EB, $>55,000$ pixels represents an EB with contractile area (s). Applying these thresholds on the image analysis data of the area of contractility, allowed for conversion of the data into 'fraction contractility'. Exposure of the cells to 6-Aminonicotinamide, Boric Acid, and Valproic Acid resulted in a dose-response relationship between the concentration of test compound and the fraction of contractility, as seen in Figs. 3-5A. Exposure to Penicillin G did not result in a clear relationship, in concordance with the findings after manual assessment (Fig. $6 \mathrm{~A}$ ). The $\mathrm{EC}_{50}$ values $(\mu \mathrm{M})$ and corresponding $\mathrm{REP}_{\text {automated }}$ values are presented in Table 2 (right panel). Ranking the compounds according to their $\mathrm{REP}_{\text {automated }}$ values resulted in the following outcome: 6-Aminonicotinamide (1) $>$ Valproic Acid $(0.008-0.013)>$ Boric acid $(0.005-0.002)>$ Penicillin $G$ (0.00001). 
A

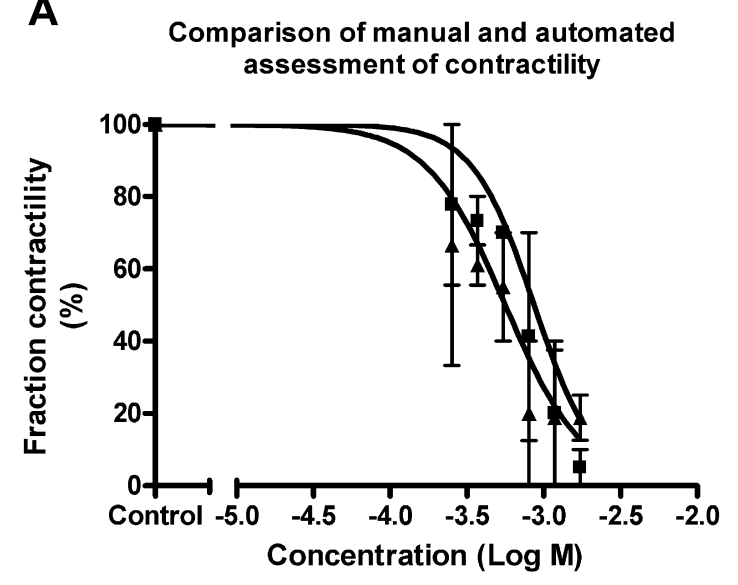

- Valproic Acid manual assessment

- Valproic Acid automated assessment

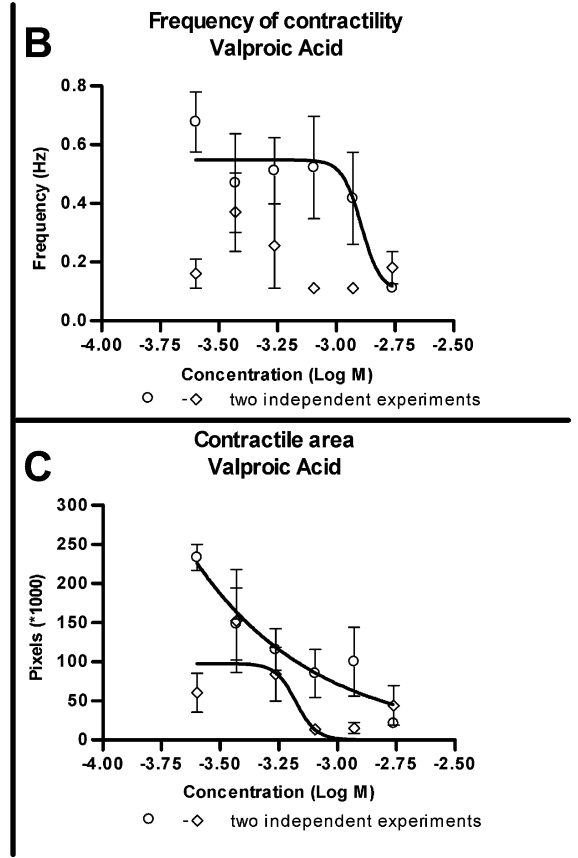

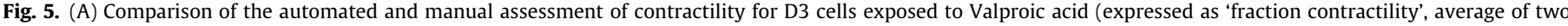

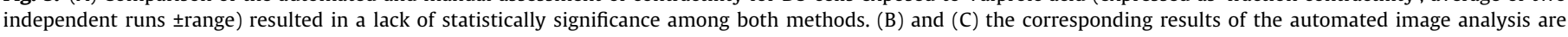

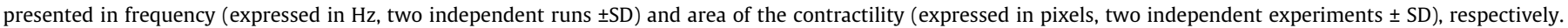

A

\section{Comparison of manual and automated assessment of contractility}

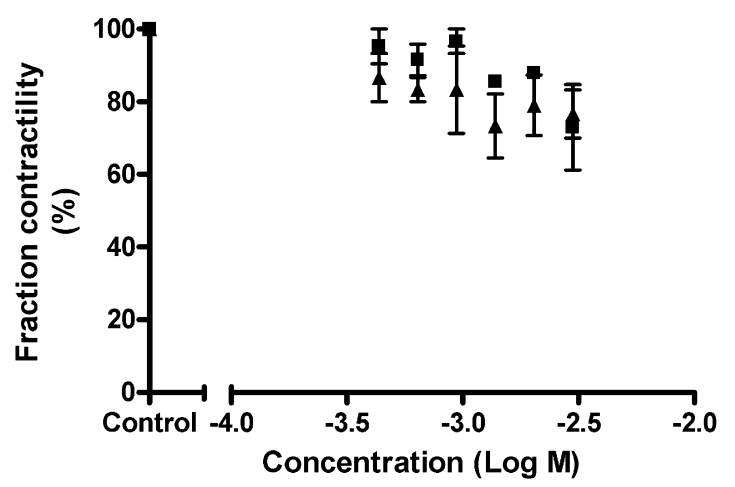

- Penicillin $\mathrm{G}$ manual assessment

- Penicillin G automated assessment

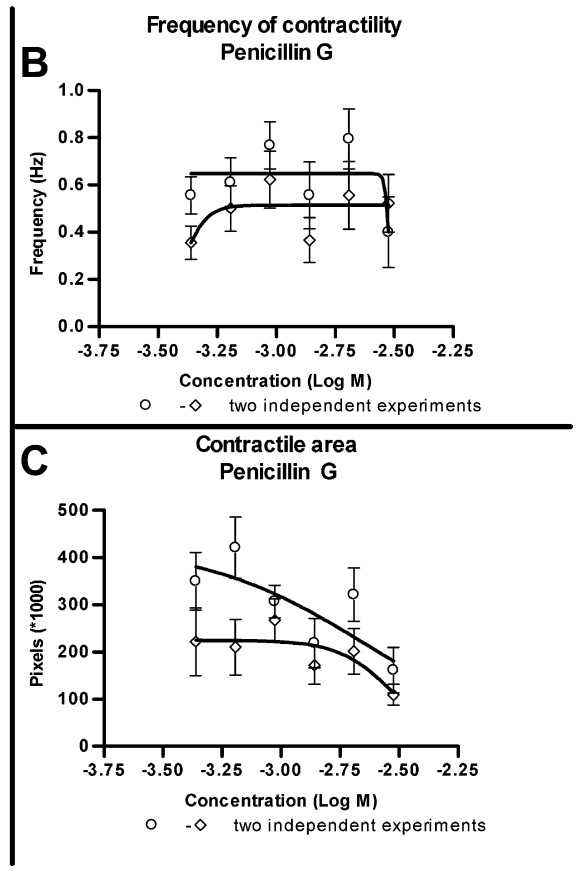

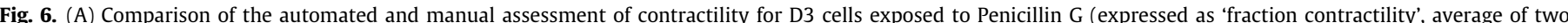

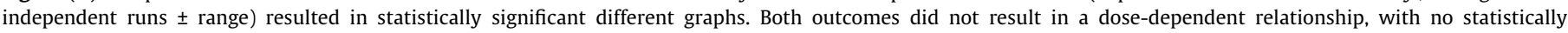

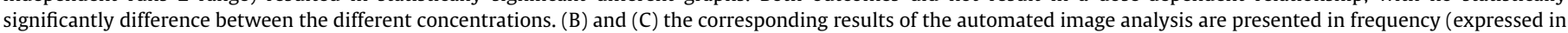
$\mathrm{Hz}$, two independent runs $\pm \mathrm{SD}$ ) and area of the contractility (expressed in pixels, two independent experiments \pm SD), respectively.

\subsection{Comparison of the manual and automated assessment of contractility}

When the outcome of the manual and automated method of assessment (in 'fraction contractility') were compared with an extra sum-of-squares F-test ( $p<0.05$; GraphPad Software, San Diego, CA, USA), no statistically significant difference was observed be- tween the data sets. After exposure to 6-Aminonicotinamide, the $\mathrm{EC}_{50}$ values of both 'fraction contractility' (based on the area of contractility with fixed thresholds for empty wells and non-contractile cells, Table 2), contractile area, and frequency (Table 3) are in the same order of magnitude: $5.35-3.5 \mu \mathrm{M}, 6.53-4.5 \mu \mathrm{M}$, and 6.75-4.32 $\mu \mathrm{M}$, respectively. The contractile areas reduce with increasing concentration of test compound (Fig. 3C), combined 
Table 3

The inhibition of differentiation of D3 cells into cardiomyocytes after exposure to test compounds was assessed with an automated method, and the area (on the left) and frequency of the contractility (on the right) computed

\begin{tabular}{lll}
\hline Compound & Contractile area & Frequency of contractility \\
\cline { 2 - 3 } & $\mathrm{EC}_{50}(\mu \mathrm{M})$ & $\mathrm{EC}_{50}(\mu \mathrm{M})$ \\
\hline 6-Aminonicotinamide (6AN) & $6.53-4.50$ & $6.75-4.32$ \\
Valproic acid & nd-665 & nd-1278 \\
Boric acid & $1464-1749$ & $1002-1718$ \\
Penicillin G & nd-2320 & nd-nd
\end{tabular}

The data are presented as $\mathrm{EC}_{50}(\mu \mathrm{M})$ of two independent experiments (nd: not detectable).

with a less frequent contraction (Fig. 3B). However, the observed variability in both the area (Fig. $3 \mathrm{C}$ ) and frequency of contractility (Fig. 3B) between the two individual runs are large.

After exposure to Boric acid, the computed contractile area and frequency (Fig. $4 \mathrm{~B}, \mathrm{C}$ ) resulted in a similar variability between the two independent runs. However, as observed after exposure to 6Aminonicotinamide, the obtained $\mathrm{EC}_{50}$ values were comparable with the data converted to 'fraction contractility' (Tables 2 and 3 ). The decrease in contractile area is concordant with a decreased frequency (Fig. 4B and C).

Exposure to Valproic acid did not result in a dose-response relationship for the area and frequency of contractility in one of the two runs (Fig. 5B and C). The data is difficult to interpret due to large variability. The obtained $\mathrm{EC}_{50}$ value for the frequency of contraction is almost double compared to the $\mathrm{EC}_{50}$ value for the contractile area: 1275 and $665 \mu \mathrm{M}$, respectively.

Exposure of the cells to the non-embryotoxic compound Penicillin $G$ did not result in a dose-response relationship when the data was converted to 'fraction contractility', neither for the manual nor for the automated assessment (Fig. 6A). The frequency of contraction did not decrease with increasing dose (Fig. 6B), while there does appear to be an inclination for reduction of the contractile area (Fig. 6C)(not statistically significant).

\section{Discussion and conclusion}

The embryonic stem cell test (EST) is proposed as a tool to detect embryotoxicity early in the development of chemical compounds and pharmaceutical candidates. Developmental toxicity assessment without the use of laboratory animals is a necessary amendment to the screening procedure.

However, the current experimental procedure of the EST with an outcome based on visual assessment of the contractility is time-consuming. Previously, the throughput of the EST was increased by utilisation of a low attachment 96-well plate that allows for the formation of embryonic bodies (EBs) (Peters et al., 2008). This resulted in a cost saving by a decreased amount of test compound and medium required for the assay to approximately $15 \%$ that of the ECVAM-validated EST.

In order to improve upon the time-consuming manual assessment of contractility, a method is proposed to automate the assay. Methods to quantify the contractility of cardiomyocytes can involve singular cells or micro-electrode assays (Reppel et al., 2004; Garcia-Webb et al., 2007). Higher throughput is offered by spiked cell cultures in which for instance calcium fluctuations can be measured, with or without incorporation of fluorescent markers (Sauer et al., 1998; Dolnikov et al., 2006). However, these methods are not suitable as a screening tool for high throughput analysis. The same applies for quantification of mRNA expression or FACS analysis as a tool to detect and quantify the embryotoxic potential of test compounds (Bigot et al., 1999) reviewed by (Buesen et al., 2004). Furthermore, the functional capacity of the cells to contract is not taken into account with these methods.
In the current study, automated video recording was assessed as a tool to quantify the outcome of the EST in the 96-well format, ensuring an even higher throughput of the assay. The automated image analysis compiled data regarding the area and frequency of contractility of a given EB over a designated time-period for each well. However, while there does seem to be a cyclic contraction in each singular EB, the observed variability between EBs exposed to the same concentration of test compound was large (Fig. 2). Furthermore, the area of contraction varied at each time-point, both in localisation and size (Fig. 1).

Similar observations were previously made by Dolnikov and colleagues, who reported on the irregular 3D structure of the EBs. The fact that the contractile cells do not shorten along a single axis of contraction made them conclude that their image recording is not linearly indicative for the force of contraction (Dolnikov et al., 2006). As presented in Fig. 2, a tendency to cyclic contraction was observed, as computed by the BEA software. The current in vitro system does not distinguish between differences in diastolic and systolic function, and can therefore not compute a true rhythmic pulse, opposite to adult cardiomyocytes. Figs. 3-6B and $\mathrm{C}$ are indicative that the computed data concerning the area and frequency of contractility are difficult to interpret.

However, the observed relationship might be a manner in which false-negative outcomes in the EST can be excluded. Compounds that do not appear to influence the cardiomyocyte differentiation and are negative when the data are compiled as 'fraction contractility', might still affect the area and frequency contractility of the cardiomyocytes. Nevertheless, the observed variability among test runs will remain a difficult factor when reviewing these kind of data.

In the adult heart, cardiomyocytes increase their contraction force under the influence of stress factors, to increase the cardiac output (hypertrophy). Dolnikov and colleagues reported a negative force-frequency relation in the differentiated embryonic cardiomyocytes. In other words, a reduction in the amplitude of the contraction is expected in the EST. As seen in Figs. 3-6B, there is an inclination that the decreased fraction contractility was accompanied by a decreased frequency of contraction.

With the low-attachment 96-well plates, occasional attachment of the cells to the plastic was observed, eliminating those EBs from further use in the assay. Consequently, this resulted in empty wells when the EBs were transferred to a tissue-culture treated 96-well plate on the fifth day of the experiment. Besides the occasional attached cells, EBs could also have been inadvertently left behind in the original well plate when they were transferred to a second well plate. When the data of the contractile area was depicted as 'fraction contractility', there was a need to include a threshold for these empty wells ( $<1500$ pixels). In the current setup, the study was considered acceptable when a minimum of eight EBs was present for each concentration of the test compounds (Peters et al., 2008). By now, there are alternatives to the low attachment plates used in the current studies, that have a similar or even better capacity to allow EB formation without attachment of the cells to the wells: Corning (ref 7007) 96-well ultra low attachment plates (round bottom), and Cellseed Hydrocell 96-well plates (round bottom).

After the threshold was set to distinguish contractile from noncontractile EBs for the automated image analysis, both manual and automated methods were presented as 'fraction contractile EBs'. The automated image analysis of the contractility proved comparable to the manual assessment, with no statistically significant difference observed $(p<0.05)$.

When the data presented in Table 2 were compared to previously reported outcomes, the $\mathrm{EC}_{50}$ values for 6-Aminonicotinamide and Valproic Acid in the current study are slightly higher than the range previously reported: $3.5-5.92 \mu \mathrm{M}$ (lowest and highest observed value of both manual and automated assessment in the current study) compared to $2.33 \pm 1.18 \mu \mathrm{M}$ for 6-Aminonicotinamide, 
and $268.9-867.3 \mu \mathrm{M}$ compared to $270.5 \pm 121.4 \mu \mathrm{M}$ for Valproic acid (Peters et al., 2008). Boric acid resulted in a lower $\mathrm{EC}_{50}$ value compared to previous experiments: $1196-1672 \mu \mathrm{M}$ compared to $1754 \pm 53.67 \mu \mathrm{M}$ (Peters et al., 2008). The observed differences are considered due to inter-experimental diversity. After exposure of the cells to Penicillin G, both in the current study with different methods of assessment as well as in the previous reported data, no $\mathrm{EC}_{50}$ value could be computed for the negative control Penicillin G.

Ranking the compounds according to their REP values was not influenced by these slightly different $\mathrm{EC}_{50}$ values. The rank-order resulted in the same hierarchy; 6-Aminonicotinamide (1) > Valproic Acid (range from 0.007-0.013 in the current study, compared to $0.011 \pm 0.003$ as previously reported) > Boric acid (range from $0.002-0.005$ in the current study, compared to $0.0014 \pm 0.001$ as previously reported) > Penicillin G (0.00001).

In both the ECVAM-validation study as well as additional literature, the test compounds are presented in three categories of developmentally toxic potency, being non, moderate and strong embryotoxic (Smith et al., 1983; Schwetz and Harris, 1993; Spielmann et al., 2001; Brown, 2002; Genschow et al., 2002, 2004). In the current study, the outcome is presented in a continuous table, with test compounds ranked by their embryotoxic potential, expressed as relative embryotoxic potential (REP; Table 2). Since the REP values are expressed relative to the outcome of the positive control 6-Aminonicotinamide of that individual experiment, changes in cell growth among experiments e.g. are taken into account. However, no unambiguous threshold can be set for developmental toxicity based on these REP values. Clear nonembryotoxic compounds such as penicillin G (Fig. 6) are identified, but the applicability domain of the EST is not transparent. Receptor mediated pharmaceuticals with known in vivo activity appear to be mis-classified (Paquette et al., 2008). The applicability domain of the EST should be further investigated since the use of the EST as an in vitro screening tool, in either 24- or 96-well format, depends on its reliability to identify developmental toxic test compounds.

Automated image analysis allows for a less time-consuming hands-on practical approach. The outcome is an objective measurement of the effect of test compounds on the differentiation of embryonic stem cells, and can be stored for later analysis. Possible observer bias, as might occur with visual analysis of the contractility, is avoided by the automation.

In order to establish a predictive relationship between the area and frequency of contractility and to evaluate possible false-negative responses, more data needs to be generated and analysed with the automated image analysis method and BEA software. For future research, the system might be complemented with fluorescent dyes to assess the influence of test compounds on the intracellular calcium concentrations (Viatchenko-Karpinski et al., 1999; Kapur et al., 2006). As suggested by Schmidt (Schmidt et al., 2001), the incorporation of markers to assess multiple cell types within the heterogeneous population of differentiated embryonic stem cells allows for a more complete picture of the effects of test compounds. Furthermore, staining of the cells with subsequent automated analysis could offer additional possibilities for assessment of a variety of markers such as cytotoxicity, morphology, or cell cycle (Walmod et al., 2002). Additionally, the auto-focus step will be accompanied by automated localisation of the embryonic body in future experiments. This can occur by applying a density threshold, since the centre of the EB contains the most dense cell population in the well plate.

\section{Conflict of interest statement}

None declared.

\section{Acknowledgement}

The studies described were partly performed with a grant from Johnson and Johnson Corporate Office of Science and Technology (COSAT). We would like to thank Miroslav Cik for all his efforts with the collaborations involved. Ruben Capiau and Margino Steemans are acknowledged for their help and technical assistance.

\section{References}

Adler, S., Paparella, M., Pellizzer, C., Hartung, T., Bremer, S., 2005. The detection of differentiation-inducing chemicals by using green fluorescent protein expression in genetically engineered teratocarcinoma cells. Altern. Lab. Anim. 33, 91-103

Adler, S., Pellizzer, C., Hareng, L., Hartung, T., Bremer, S., 2008. First steps in establishing a developmental toxicity test method based on human embryonic stem cells. Toxicol. In Vitro 22, 200-211.

Bigot, K., De Lange, J., Archer, G., Clothier, R., Bremer, S., 1999. The relative semiquantification of mrna expression as a useful toxicological endpoint for the identi ${ }^{\circledR}$ cation of embryotoxic/teratogenic substances. Toxicol. In Vitro 13, 619623.

Bremer, S., Worth, A.P., Paparella, M., Bigot, K., Kolossov, E., Fleischmann, B.K., Hescheler, J., Balls, M., 2001. Establishment of an in vitro reporter gene assay for developmental cardiac toxicity. Toxicol. In Vitro 15, 215-223.

Brown, N.A., 2002. Selection of test chemicals for the ECVAM international validation study on in vitro embryotoxicity tests European centre for the validation of alternative methods.. Altern. Lab. Anim. 30, 177-198.

Buesen, R., Visan, A., Genschow, E., Slawik, B., Spielmann, H., Seiler, A., 2004. Trends in improving the embryonic stem cell test (EST): an overview. Altex 21, $15-22$.

De Smedt, A., Steemans, M., De Boeck, M., Peters, A.K., van der Leede, B.J., Van Goethem, F., Lampo, A., Vanparys, P., 2008. Optimisation of the cell cultivation methods in the embryonic stem cell test results in an increased differentiation potential of the cells into strong beating myocard cells. Toxicol. In Vitro. doi:10.1016/j.tiv.2008.07.003.

Dolnikov, K., Shilkrut, M., Zeevi-Levin, N., Gerecht-Nir, S., Amit, M., Danon, A., Itskovitz-Eldor, J., Binah, O., 2006. Functional properties of human embryonic stem cell-derived cardiomyocytes: intracellular Ca2+ handling and the role of sarcoplasmic reticulum in the contraction. Stem Cells 24, 236-245.

Garcia-Webb, M.G., Taberner, A.J., Hogan, N.C., Hunter, I.W., 2007. A modular instrument for exploring the mechanics of cardiac myocytes. Am. J. Physiol. Heart C.

Genschow, E., Spielmann, H., Scholz, G., Pohl, I., Seiler, A., Clemann, N., Bremer, S., Becker, K., 2004. Validation of the embryonic stem cell test in the international ECVAM validation study on three in vitro embryotoxicity tests. Altern. Lab. Anim. 32, 209-244.

Genschow, E., Spielmann, H., Scholz, G., Seiler, A., Brown, N., Piersma, A., Brady, M., Clemann, N., Huuskonen, H., Paillard, F., Bremer, S., Becker, K., 2002. The ECVAM international validation study on in vitro embryotoxicity tests: results of the definitive phase and evaluation of prediction models. European centre for the validation of alternative methods. Altern. Lab. Anim. 30, 151-176.

Honda, M., Kurisaki, A., Ohnuma, K., Okochi, H., Hamazaki, T.S., Asashima, M., 2006. $\mathrm{N}$-cadherin is a useful marker for the progenitor of cardiomyocytes differentiated from mouse ES cells in serum-free condition. Biochem. Biophys. Res. Commun. 351, 877-882.

Kapur, N., Mignery, G., Banach, K., 2006. Cell cycle dependent calcium oscillations in mouse embryonic stem cells. Am. J. Physiol. -Cell Physiol..

Paquette, J.A., Kumpf, S.W., Streck, R.D., Thomson, J.J., Chapin, R.E., Stedman, D.B., 2008. Assessment of the embryonic stem cell test and application and use in the pharmaceutical industry. Birth Defects Res. B Dev. Reprod. Toxicol. 83, 104111.

Peters, A.K., Steemans, M., Mesens, N., Hansen, E., Verheyen, G.R., Spanhaak, S., Coussement, W., and Vanparys, P., 2007. A higher throughput method to the embryonic stem cell test (EST), to detect embryotoxicity in early development. In: Proceedings of the Sixth World Congress on Alternatives \& Animal Use in the Life Sciences, pp. 673-677.

Peters, A.K., Steemans, M., Mesens, N., Hansen, E., Verheyen, G.R., Vanparys, P., 2008. Evaluation of the embryotoxic potency of compounds in a newly revised embryonic stem cell test. Toxicol. Sci.. doi:10.1093/toxsci/kfn126.

Piersma, A.H., 2004. Validation of alternative methods for developmental toxicity testing. Toxicol Lett 149, 147-153.

Piersma, A.H., Attenon, P., Bechter, R., Govers, M.J., Krafft, N., Schmid, B.P., Stadler, J., Verhoef, A., Verseil, C., 1995. Interlaboratory evaluation of embryotoxicity in the postimplantation rat embryo culture. Reprod. Toxicol. 9, 275-280.

Reppel, M., Boettinger, C., Hescheler, J., 2004. Beta-adrenergic and muscarinic modulation of human embryonic stem cell-derived cardiomyocytes. Cell Physiol. Biochem. 14, 187-196.

Rohwedel, J., Guan, K., Hegert, C., Wobus, A.M., 2001. Embryonic stem cells as an in vitro model for mutagenicity, cytotoxicity and embryotoxicity studies: present state and future prospects. Toxicol. In Vitro 15, 741-753.

Satoh, H., Ginsburg, K.S., Qing, K., Terada, H., Hayashi, H., Bers, D.M., 2000. KB-R7943 block of $\mathrm{Ca}(2+)$ influx via $\mathrm{Na}(+) / \mathrm{Ca}(2+)$ exchange does not alter twitches or 
glycoside inotropy but prevents $\mathrm{Ca}(2+)$ overload in rat ventricular myocytes. Circulation 101, 1441-1446.

Sauer, H., Hofmann, C., Wartenberg, M., Wobus, A.M., Hescheler, J., 1998. Spontaneous calcium oscillations in embryonic stem cell-derived primitive endodermal cells. Exp. Cell Res. 238, 13-22.

Schmidt, M.M., Guan, K., Wobus, A.M., 2001. Lithium influences differentiation and tissue-specific gene expression of mouse embryonic stem (ES) cells in vitro. Int. J. Dev. Biol. 45, 421-429.

Schwetz, B.A., Harris, M.W., 1993. Developmental toxicology: status of the field and contribution of the National Toxicology Program. Environ. Health Perspect 100, 269-282.

Seiler, A., Visan, A., Buesen, R., Genschow, E., Spielmann, H., 2004. Improvement of an in vitro stem cell assay for developmental toxicity: the use of molecular endpoints in the embryonic stem cell test. Reprod. Toxicol. 18, 231-240.

Smith, M.K., Kimmel, G.L., Kochhar, D.M., Shepard, T.H., Spielberg, S.P., Wilson, J.G., 1983. A selection of candidate compounds for in vitro teratogenesis test validation. Teratogen. Carcin. Mut. 3, 461-480.

Spielmann, H., Genschow, E., Scholz, G., Brown, N.A., Piersma, A.H., Brady, M., Clemann, N., Huuskonen, H., Paillard, F., Bremer, S., Becker, K., 2001. Preliminary results of the ECVAM validation study on three in vitro embryotoxicity tests. Altern. Lab. Anim. 29, 301-303.

Spielmann, H., Pohl, I., Doring, B., Liebsch, M., Moldenhauer, F., 1997. The embryonic stem cell test (EST), an in vitro embryotoxicity test using two permanent mouse cell lines; 3t3 fibroblasts and embryonic stem cells. Toxicol. In Vitro 10, 119127.

Viatchenko-Karpinski, S., Fleischmann, B.K., Liu, Q., Sauer, H., Gryshchenko, O., Ji, G.J., Hescheler, J., 1999. Intracellular Ca2+ oscillations drive spontaneous contractions in cardiomyocytes during early development. Proc. Natl. Acad. Sci. USA 96, 8259-8264.

Walmod, P.S., Berezin, A., Gallagher, H.C., Gravemann, U., Lepekhin, E.A., Belman, V., Bacon, C.L., Nau, H., Regan, C.M., Berezin, V., Bock, E., 2002. Automated in vitro screening of teratogens. Toxicol. Appl. Pharmacol. 181, 1-15.

Walmod, P.S., Gravemann, U., Nau, H., Berezin, V., Bock, E., 2004. Discriminative power of an assay for automated in vitro screening of teratogens. Toxicol. In Vitro 18, 511-525.

Zur Nieden, N.I., Kempka, G., Ahr, H.J., 2004. Molecular multiple endpoint embryonic stem cell test-a possible approach to test for the teratogenic potential of compounds. Toxicol. Appl. Pharmacol. 194, 257-269. 